Dr. Baillie says, - "It has never occurred to me to see an example of this kind; but one can the more readily believe that it may sometimes happen, as the gall-bladder does not serve any necessary purpose in the body." Meckel states that-" Notwithstanding its primitive narrowness, the gallbladder is never deficient at any period, according to our observations, as one would be led to believe from some cases where its total absence has been asserted." The above remark, however, is little in accordance with the subjoined, from the same author, in his description of the biliary passages:- " Sometimes, but rarely, a part of the biliary passages, particularly the gall-bladder, is deficient from a primitive deviation of formation, although this anomaly does not necessarily exercise an injurious influence on the health, which is less astonishing, since, according to the experiments of Herlin, the gall-bladder may be extirpated in cats without inconvenience, and is normally absent in many animals." Boulet once examined a case analogous to that I have described, and subsequently in the son of the subject found the like deficiency.

St. Martin's-lane, sept. 1847.

\section{DETAILS OF A CASE OF ABDOMINAL TUMOUR SIMULATING PREGNANCY.}

Bx JOHN CHALLICE, Esq., Bermondsey.

ON the morning of the 12th of August, 1846, a messenger came to me, breathless, saying that her mistress's daughter was dangerously ill with the cholera, and that I must come directly. On my way, the maidservant dropped several hints, at first rather obscure, afterwards of a less doubtful character. "She hoped it was nothing worse than the cholera" and persons who had their living to get " could see, although they daren't say nothing. These inmendoes, conveyed in a tone and manner not to be mistaken, led me to the conclusion, that on my arrival $I$ should find the patient in the pangs of parturition. When I arrived, I saw a young female in bed, lying on her right side, with her face buried in the pillow, and the knees drawn up towards the abdomen. She seemed to be in pain, but was sullen, and refused to answer any questions. The mother told me that she had been vomiting, and complaining of pains in the loins, with a constant desire to pass water, and that for the last five or six months she had observed a change in her daughter--the appetite capricious, temper irritable, and on several occasions she had been surprised in tears; notwithstanding, she denied being ill, and continued to perform her domestic duties. These facts seemed confirmatory of the servant's suspicions, and with almost a conviction in my mind of the condition of the girl, I placed my hand upon the abdomen: it was tense and swollen, and a movement like that of a living fotus was distinctly felt; I then listened and detected a loud and quick pulsation.

The presence of these symptoms induced me to pronounce the patient pregnant. No suspicion had entered the mother's mind: she was an only daughter, and bore an excellent character. However, she did not deny the fact, but after a distressing burst of grief, and a pitiable appeal for forgiveness, she confessed that her "Cousin John had had connexion with her once, and only once, about six months before, a few days previous to his departure from England. Being unwilling to aggravate the sufferings $I$ then witnessed, by what appeared nnnecessary inquiries, or to disturb the patient by further and more careful examination, considering the case quite decisive, I contented myself with prescribing some simple remedy for relieving the sickness and pain. The next day there was a great improvement in the condition of the patient; the fear of discovery no loncer agitated her, and she had been forgiven. Up to this period she had so contrived to compress her figure, that no increase in her bullk was perceptible when dressed, although her size was quite that of the sixth month of gestation when undressed. Now that this cruel mental and physical restraint no longer tormented her, she suffered less from pain and sickness, became less sullen, and more communicative.

It appears that the connexion took place, after prolonged resistance, just previous to the usual period of menstruation; that up to that time there had never been the least irregularity of this function during the three years she had menstruated.

She was greatly alarmed at the absence of the accustomed appearances at the usual time, and did not feel well in health, although slie had no marked symptoms; a general sense of unersiness, with pains in the loins, and an occasional slight feeling of sickness and loss of appetite, were felt. When the next period came round, she was pleased at finding herself " unwell," but only to about half the usual extent; menstrua- tion had continued regularly up to the time I saw her; on each occasion, however, more and more scantily. The ab. domen had gone on gradually increasing in bulk, and abont five months after the connexion the patient was conscious of a movement and pulsation in the abdomen, and believed herself pregnant. The breasts were small, and marked with an indistinct areola; around the eyes and mouth there were dark circles, and her mother says she is much fallen away in flesh. Previous to this unfortunate occurrence, the patient not only enjoyed good health, but was remarkable for strength, endurance, and activity, inclined to enbonpoint, full of life and spirits, and in her nineteenth year.

During the next month or six weeks, I saw the patient occasionally. She complained of no urgent symptom, walked out now and then, had a good appetite and digestion, with sometimes slight irritability of the bladder, and irregularity of the bowels. The gradual increase in size still went on, and the mother (who now slept with her daughter) said that the movement of the child continued. The patient complained of its violence when in bed, and also began to suffer from lumbar pains, and constant irritation of the labia, which was much increased when she drank beer, wine, or spirits. And so the case went on.

When the ninth calendar month had nearly expired since the connexion, I became much interested in the case, thinking it one in which the period of gestation could be accurately ascertained.

On the evening of the expiration of the ninth month, I received the expected message, with an urgent request to hasten, as very strong labour had come on. When I arrived, the patient was standing at the foot of the bed, grasping the bedpost, and evidently suffering from pain, although not of a violent character. There was an interval of about ten minutes in the pains, during which she walked about the room, having a very anxious and haggard look.

After a good deal of persuasion, she consented to an examination per vaginam, which seemed to cause excessive pain, as she screamed violently, and exclaimed that she was being murdered. At the time, I thought the patient hysterical, but was much surprised at the narrow constricted condition of the vagina, and the presence of the hymen nearly perfect; the agony, however, produced by the examination seemed so intolerable, that the patient, by a sudden and violent effort, threw herself from me, declaring that I should torment her no more.

Finding that the pains were weak and ineffectual, and at longer intervals, and feeling assured, from the condition of the parts, that immediate labour was not at hand, I gave twenty minims of opium, and left, directing a full dose of castor oil to be given in a few hours. During the night she slept well; the oil acted freely in the morning; and the next day passed over without pain or any inconvenience, the patient having a good appetite and being in better spirits. About eleven o'clock at night the pains returned with increased violence, and I found her straining and bearing-down at the bed-post. An old-experienced nurse declared "that the pains were quite strong enough, with assistance, to bring the child into the world." The mother states, that during the night she had placed her hand on her daughter's stomach, and felt the child move vigorously.

In the intervals of pain, the patient walked about the room and was cheerful, except expressing what seemed an unreasonable horror at any examination. The pains commenced in the abdomen, and then extended round to the loins, came on reoularly every ten or fifteen minutes, and were marked with all the characteristics of labour in its first stage.

The extreme excitement and dread which the patient evinced when the necessity for an examination was impressed upon her, induced me to waive it, although I was anxious to ascertain the real condition of affairs. It would be useless to detail the diurnal symptoms-suffice it, that a week passed over, and matters remained apparently without alteration, either one way or the other. I may here state, that menstruation did not take place at this period. Doubts now first began to arise in my mind about the nature of the case, and when nine calendar months from the departure of her cousin had expired, I became very anxious about it. It was at this stage that Dr. Lever was consulted. After a careful and thorongh external and internal examination, this gentleman, justly famous for his skill and tact in diagnosis, having the history of the case before him, came to the conclusion that it was " extra-nterine impregnation." At that time her physical condition was as follows:- Countenance pale - an anxious expression; eyes rather sunken; nose pinched; breasts some 
what flaccid; abdomen the size of mature pregnancy, if not larger; pulse never less than 100; the tongue clean, but morbidly red; bowels sometimes costive for a day or two, at other times the reverse; urine most frequently pale and copious, but on some occasions thick, scanty, and high-coloured. Over the entire abdominal region a distinct pulsation could be heard and felt; but owing to the extreme excitability of the patient, it was almost impossible to ascertain whether or not it was synchronous with the pulse. Palliative measures were adopted, and the case, now become one of painful interest, was closely watched. During the next fortnight no perceptible alteration occurred, except that the pulsation in the tumour became less distinct, and the abdomen more tense. Dr. Ferguson now visited the patient, and pronounced the abdominal pulsation to be synchronous with the heart's action, and doubted whether impregnation had taken place at all. On his recommendation, $\mathbf{I}$ punctured the abdomen with a fine "trochar," and drew off about five pints of thick grumous and offensive matter. Great relief followed the operation, only, however, temporary; for in the course of a short time the abdomen became as tense as before, and all the patient's sufferings returned. The interest, in a further detail of the symptoms of this case, here ceases, no doubt now being entertained of its character. After a second and a third tapping, the poor girl gradually got weaker and weaker, her only comfort the oblivion produced by anodynes; and on the 15th of February she died.

The day following, assisted by my friend, Mr. Druitt, we made a post-mortem examination. The upper portion of the body was extremely emaciated, but, owing to slight œedema of the lower extremities, this appearance was not general. Abdomen greatly distended, and marked by enlarged veins; it measured in circumference fifty-eight inches. About a gallon of fluid was drawn off by the trochar, previous to making a free incision, after which, nearly a pailful of brain-like matter rolled out. This had been contained in a cyst, which ex tended from the pubis to the ensiform cartilage, and from the left to the right hypochondrium; in some parts, the walls of the sac were more than an inch thick, and of a fibro-cartilaginous consistence; the anterior portion adhered firmly to the abdominal parietes, the upper being formed by the inferior surface of the liver; that organ was bathed with the contents of the sac, and became inoculated, several small cysts, filled with medullary sarcoma, having formed in its substance. There were, also, many isolated cysts, varying from the size of a hazel nut to that of a pigeon's egg, formed in the walls of the cyst; these had no connexion with each other, or communication with the general cavity. The uterus was found imbedded in the lower portion, or base of the cyst; no trace of the ovaries could be met with; the bladder was small, but not affected by disease.

The peculiar interest of this case arises from the close resemblance to the symptoms of impregnation; the development of a malignant disease, seeming, in a great measure, to be influenced by the feelings or instinct of the patient. Would the girl have died, had no connexion taken place? How far did the mental and plysical excitement act upon the origin or the progress of the disease? Or was it completely independent, and its course inevitable?

Octcber, 1817 .

THE

SPASMS OF HYDROPHOBIA TEMPORARILY

RELIEVED BY THE INHALATION OF THE VAPOUR OF SULPHURIC ETHER.

By ROBERT AILAN, Esq., Staff-Surgeon, Mauritius.

RAMJAN, an Indian boy, aged twelve, in the service of Madame Gébert, was bitten on the right ankle by a rabid dog, in May, 1846: there were four incisions made by the dog's teeth, and the part was bare when injured. The sesquichloride of antimony was applied to the wounds in a few hours after, by some druggist, and the dog was shot.

About the end of May, 1847, he felt pain in the right knee, with a general uneasiness; but these symptoms passed away, and on the 8th of June following he beran to complain of pain in the throat, and a difficulty in swallowing liquids. Dr. Salesse, an experienced practitioner, was called to the patient, on the evening of the $9 \mathrm{th}$, and he invited me, along with several other medical brethren, to see the boy, on the $10 \mathrm{th}$, at eleven o'clock A.M., when we found him sitting up in bed, with a wild expression of countenance, cold perspiration, and frequent spitting of a thick saliva; pulse gnick and feeble; bowels constipated. When asked if he had been bitten, he pointed out the part, and remarked that it was completely cured. Spasms were instantly induced by opening the window, blowing on his face, or making him attempt to drink. He had not slept since the $8 \mathrm{th}$, and had eaten nothing during the last twenty-eight hours.

We unanimously recommended that the inhalation of the vapour of ether should be tried; and the apparatus employed was merely a bottle with an opening made in its bottom, and a sponge, saturated with an ounce of ether, inserted, as sug. gested by my brother, in THe LanceT of February 6 th, 1847, page 163.

With a great deal of perstasion, we prevailed on him to inhale a little, but he constantly removed the bottle from his mouth, saying he would rather die than take any kind of medicine, and requested to be left alone. We now determined to make him inhale by force, as the spasms had been considerably calmed; but the slight effects produced wore off almost immediately, from the imperfect manner in which the process was carried on. Half an ounce of ether was added to the sponge, and the apparatus held to his mouth, the nostrils being closed, while his struggles were overcome by manual force. In three minutes from the commencement of this second trial he became insensible to pinching, and remained so about six minutes, the pulse being 120, and extremely feeble. He soon began to talk, at first incoherently, but in a short time asked for " rice, currie, and chattny, as he had not eaten for two days." He ate a large quantity of these with avidity, drank a little claret, and then called for water, with which he rinsed his moath and washed his hands, after the manner of his countrymen. We now blew on his face several times, without inducing spasm, and he laughed when the rays of light were thrown on it by a mirror. At half-past one p.M. we made him inhale (by using a little force) until he became drowsy. At half-past two he ate part of an orange, and inhaled without opposition. At four we found him again restless, with a wild expression of countenance, and a cold perspiration. His mother then said she would not allow him to inhale the ether any more, as it would kill him, and that his father had tied a charm round his wrist, which would effect a cure by next morning.

We all agreed upon the propriety of repeating the inhalation of ether, as the benefit derived from it during the day had been so evident, and the process was therefore carried. through by manual force, Although he used his utmost endeavour's to defeat our design, he was completely stupified in four minutes, and was then attacked with a strong convulsion of about a minute's duration; after which, the pulse could scarcely be felt, and the respirations were 28 in a minute. While in this state, an enema, containing an ounce of turpentine, was thrown up, which was ejected with great force in a quarter of an hour after, but brought away no feces. At six in the evening he again ate some rice and curry; shortly after which, we made him inhale the ether by force, its usual effects being produced at the end of six minutes. The pulse could not be felt at the wrist for a few seconds, but ho slowly revived, and remained comparatively quiet for an hour. Another enema was now thrown up; after which, he voided a little urine, and a great quantity of flatus from the rectum, but no frees; pulse varying from 120 to 140 , sometimes remarliably weak and thready. At eight he became very restless; and at night we brought the ether apparatus to him, but he broke the bottle. We were therefore under the necessity of applying a sponge, soaked with etlier, to his mouth, while the nos trils were kept closed, and in two minutes he became perfectly calm, the simple sponge liaving produced the same effect in as short a time as the most complicated inhaling apparatus could have done - with a great expenditure of ether, however.

In the evening he called frequently for water, a lithle of which he swallowed with difficulty, and threw a quantity intentionally over his bocy. Since six P.x., we observed that the penis remained half erect," and he continued to pull it with his hands; the abdomen was swollen, and a great deal of wind passed from the intestincs each time he was etherized. There was also opisthotonos, with frequent ineffectual attempts to romit; and he complained of pain in the throat and chest. After ten o'clock he repeatedly called for the ether-sponge, and said it relieved him; the pulse conld not be felt at the wrist, and in the carotios it was feeble and irregular. $\mathrm{He}_{\mathrm{e}}$ continued in this state minl half.past one on the morning of the 11th, when he expires.

Examination, eleven hours after deals.-Countenance placid, and abdomen slightly tumid. A few patches of extravasated

* Mead has also remarked the same condition: vide "A Mechanical Account of Poisons." Iondon, thiri edition, $1 / 15$, p. 133 . 